\title{
Hygienic aspects of wearing shoes
}

\author{
Kolomaznik K* and Pecha J
}

Faculty of Applied Informatics, Tomas Bata University in Zlin, Czech Republic

\begin{abstract}
The paper deals with the quantitative description of microclimate in the inner volume of shoes. Unsuitable microclimate conditions may damage foot skin. Isothermal quantitative model of shoe microclimate based on mass balance is presented. Model takes into account moisture produced by foot skin, accumulation of moisture in the inner shoe volume and its transport through shoe upper material. Model calculations of microclimate were done for several different transport properties of the shoe material. In addition, the problematic of leather tanned with chromium compounds it discussed in connection with potential presence of toxic hexavalent chromium compounds and other compounds which may be present in the shoe upper material and which may affect human health. The positive role of wearing socks which may act as a barrier for transport of harmful compounds to the foot skin and also act as a regulator of inner shoe microclimate is emphasized.
\end{abstract}

\section{List of symbols}

$\dot{m}$ : density of moisture flow $\left[\mathrm{kg} \mathrm{m}^{-2} \mathrm{~s}^{-1}\right] ; S$ : surface both skin and inner shoe material $\left[\mathrm{m}^{2}\right] ; V$ : free inner volume of shoes $\left[\mathrm{m}^{3}\right] ; c$ : concentration of moisture in the inner volume $\left[\mathrm{kg} \mathrm{m}^{3}\right] ; c_{o}$ : moisture concentration outside of shoes $\left[\mathrm{kg} \mathrm{m}^{3}\right] ; c_{n}$ : equilibrium moisture concentration $[\mathrm{kg}$ $\left.\mathrm{m}^{3}\right] ; \tau$ : time [s]; $D$ : effective diffusion coefficient steam in shoe upper material $\left[\mathrm{m}^{2} \mathrm{~s}^{-1}\right] ; x$ : coordinate (position in upper shoe thickness) $[\mathrm{m}]$; $\delta$ : thickness of upper shoe material $[\mathrm{m}] ; p_{n}$ : equilibrium pressure of water vapor at specific temperature $[\mathrm{Pa}] ; \phi$ : relative moisture [1]

$R$ : universal gas constant $8314 \mathrm{~J} \mathrm{kmol}^{-1} \mathrm{~K}^{-1}\left[\mathrm{~J} \mathrm{kmol}^{-1} \mathrm{~K}^{-1}\right]$; $T$ : absolute temperature $[\mathrm{K}]$; $A$ : Langmuir isotherm adsorption constant [1]; $B$ : Langmuir isotherm adsorption constant $\left[\mathrm{m}^{3} \mathrm{~kg}^{-1}\right] ; K$ : Langmuir isotherm adsorption constant [1]

\section{Introduction}

The main function of shoes is to protect human feet from mechanical damage, unfavorable weather conditions and last but not least from potential damage caused by the surroundings.

Apart from the above mentioned characteristics there is also a significant factor related to fashion. Ideal shoes should meet all the mentioned needs and cannot negatively affect the feeling of comfort and medical harmlessness during their practical use - wearing. This is, however, the ideal case but the reality is different. Shoes which are not chosen properly can damage human health significantly, often with permanent consequences. Negative aspects of wearing improper shoes can be divided into two main categories - orthopedic damage, which in many cases results in permanent deformation caused by damage of supporting bones of legs or various apophysics. Danger of orthopedic damage is especially high among children from an early age. Because of this it is necessary to pay close attention while buying children's shoes and avoid goods of low quality (usually dubiously low priced). Other possible damage is connected to dermatological problematics during which skin is damaged. Orthopedic damage can be avoided relatively easy while shopping - as a very simple and approximate rule - if shoes do not fit, we should not buy them. Dermatological damage of skin in many cases appears after some time, or sometimes even after guarantee and for this reason we will deal with dermatological problems in detail.
Relatively simple example seems to be shoes made from non-toxic material - leather, textile or plastic. Even non-toxic material from which shoes are made can cause dermatological problems connected to microclimate in inner volume, which is the volume bounded by the inner surface of the footwear and surface of foot skin. Inappropriate microclimate, dampness and temperature can create favorable conditions for skin or nail mold in inner volume, or possibly fungus and yeast diseases of skin and nails. Curing the mentioned problems is very long and expensive, not to mention the discomfort it causes.

\section{Quantitative description of the microclimate in the inner volume of shoes}

The surface of skin is a source of moisture and heat that is accumulated in the inner volume of shoes and is transported through the upper shoe material. The quantitative model describes nonstationary heat and mass balance, the solution of which is the time dependence of concentration and temperature, i.e. the microclimatic conditions in the inner volume of shoes. For formation of skin diseases, moisture is of prime importance, and for this reason we will discuss an isothermal model. The exact mathematical model [1], of course, is of great importance, but due to its complexity and for practical reasons, we will present an approximate model that is relatively simple and whose solution does not cause great difficulties. In the approximate model, the nonlinear concentration profile in the upper material is replaced by the linear concentration profile in the shoe upper material. This is, then, a quasi-stationary model:

$$
\begin{aligned}
& \dot{m} S=V \frac{\partial c(\tau)}{\partial \tau}-D S \frac{\partial c(0, \tau)}{\partial x} \\
& \text { moisture source }=\text { accumulation of moisture, transport of moisture } \\
& \text { (skin surface) } \quad=(\text { internal footwear volume), (upper part of a shoe) }
\end{aligned}
$$

Correspondence to: Kolomaznik K, Faculty of Applied Informatics, Tomas Bata University in Zlin, Czech Republic, Tel: +420 57-603-5256; E-mail: kolomaznik@fai.utb.cz

Key words: shoes, inner volume, microclimate, dermatology, chromium VI, sock, quasi-stationary model

Received: April 02, 2017; Accepted: April 24, 2017; Published: April 29, 2017 
where $x$ stands for the position in the shoe upper material ( $x=0$ is the inner surface of the shoe upper material). By taking into account that at the beginning the moisture content in the shoe is equal to zero (i.e. the initial condition) and substituting of concentration gradient on the shoe upper material inner surface with difference of moisture concentration in the inner volume and outside (expression 2 - quasi stationary case) we will obtain final expression (3) by integration of equation (1).

$$
\begin{aligned}
& -\frac{\partial c(0, \tau)}{\partial x} \approx \frac{c(\tau)-c_{0}}{\delta} \\
& c(\tau)=\frac{\dot{m} \delta+D c_{0}}{D}\left[1-\exp \left(-\frac{D S}{\delta V} \tau\right)\right]
\end{aligned}
$$

For the concentration of humidity in the surroundings and its equilibrium concentration in the internal volume we use the formula:

$$
c=\frac{p_{n} \varphi M}{R T}
$$

\section{Microclimate mathematical modelling}

\section{The upper part without surface modification}

Input data: Thickness of the upper material delta $=2.10^{-3} \mathrm{~m}$, moisture (sweat) production rate [2] $\dot{m}=$ (8.3 small, 16.1 medium, 21.7 heavyload). $10^{-5} \mathrm{~kg} \mathrm{~m}^{-2} \mathrm{~s}^{-1}$, diffusion coefficient of moisture in the upper material [1] $D=1,3.10^{-5} \mathrm{~m}^{2} \mathrm{~s}^{-1}$, skin and shoe surface $S=0.01 \mathrm{~m}^{2}$, internal volume $V=10^{-4} \mathrm{~m}^{3}$, internal temperature $35^{\circ} \mathrm{C}$, outside temperature $20^{\circ} \mathrm{C}$, ambient relative humidity $0.6(60 \%)$, ambient humidity concentration $0.01 \mathrm{~kg} \mathrm{~m}^{-3}$, concentration of saturated water vapors in the inner volume at $35^{\circ} \mathrm{C} c_{n}=0.04 \mathrm{~kg} \mathrm{~m}^{-3}$ (i.e. equilibrium humidity). Relative humidity in the internal volume is defined as the ratio of the moisture concentration calculated according to the relation (3) to the saturated water vapor concentration $c_{n}$. (Equilibrium humidity is given by the partial pressure of saturated water vapor at a given temperature - relationship (4)) The dependence of relative humidity concentration in the inner volume of a shoe is shown in figure 1 .

\section{The upper part with surface modification}

Typically, the surface treatment is achieved by applying a

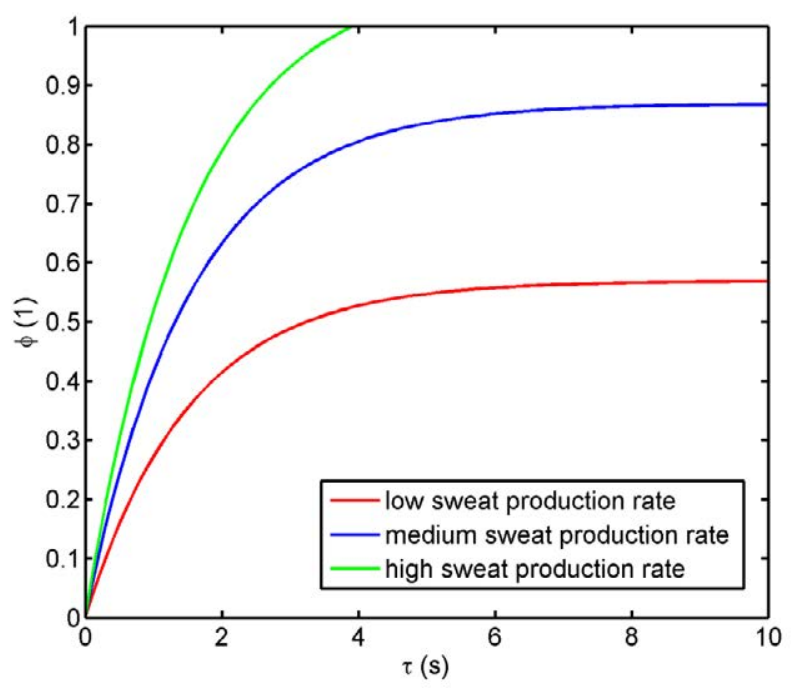

Figure 1. Time dependence of relative humidity concentration in the inner volume of a shoe. polyurethane or polyacrylate resin to form a film with a thickness of 1-10 microns. For quantitative description, it is useful to compare the diffusion resistance of both layers, leather and polymer. The diffusion resistance is defined as the ratio of the thickness of the individual layer to the diffusion coefficient value (i.e. $\delta / D$ ). The diffusion coefficient of water vapor through the polymer layer is in the order of $10^{-9} \mathrm{~m}^{2} \mathrm{~s}^{-1}$. In the case of the leather layer, the value of the diffusion resistance is $150 \mathrm{~s} \mathrm{~m}^{-1}$, with the polymer coating $1000-10000 \mathrm{~s} \mathrm{~m}^{-1}$. It is therefore possible to neglect the diffusion resistance of the leather and to deal only with the diffusion of water vapor through the polymer layer. The situation is illustrated in figure 2. The same data as in figure 1 was used for the model calculation with the difference that the thickness of the polymer layer was 10 microns and a diffusion coefficient of $10^{-9} \mathrm{~m}^{2} \mathrm{~s}^{-1}$ was used.

If we compare the results of these cases, it is more convenient to use a shoe without surface modification, which is practically met with textile material. The textile upper, despite its advantages, is not a versatile solution and therefore, in the case of leather material where the surface modification is required, the appropriate microclimate is achieved by using a sorption material with a high sorption capacity for water vapor. Experimentally, the sorption capacity is determined from the sorption isotherms, which is the dependence of the equilibrium sorbed (bound) moisture on sorption material (sorbent) to the equilibrium concentration of unbound moisture. The adsorption isotherm (Langmuir) is shown in figure 3.

A quantitative description of the graphical presentation, Langmuir isotherm is as follows:

$$
c_{A}=\frac{A c}{1+B c}
$$

For the practical estimation of the microclimate the linear part of Langmuir isotherm is sufficient.

$$
c_{A}=K c
$$

We include accumulation of sorbed moisture into balance equation (1), again for the quasi-stationary case:

$$
\dot{m} S=V(K+1) \frac{d c(\tau)}{d \tau}+D S \frac{c(\tau)-c_{0}}{\delta}
$$

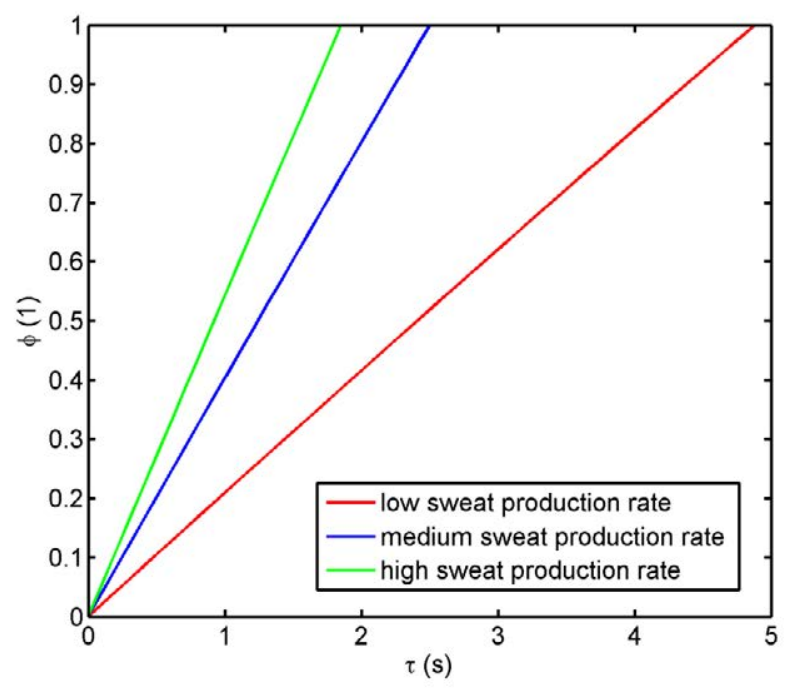

Figure 2. Time dependence of relative humidity concentration in the inner volume of a shoe, shoe upper made of leather with polymer surface treatment. 
By solving the differential equation (7) we obtain:

$$
c(\tau)=\frac{\dot{m} \delta+D c_{0}}{D}\left[1-\exp \left(-\frac{D S}{\delta V(K+1)} \tau\right)\right]
$$

An example of a model calculation for a microclimate of a shoe with a surface modification and containing a sorption material with a linear adsorption constant $K=1000$ is shown in figure 4 . Other input data is the same as in figure 2.

\section{Protection against potential poisons}

The leather includes the tanning industry. The tanning industry products are input raw materials for shoe industry, leather goods industry and textile industry. The main raw materials for the tanning industry are raw skins of cattle and game, which turn by a set of tanning operations into leather. The most important operation of the process where raw hide turns into leather is the reaction when hide is stabilized by the chemical agents. Almost $80 \%$ of hide is treating by basic complex chromic (Cr III) salts. Chromium in the leather is bound by strong

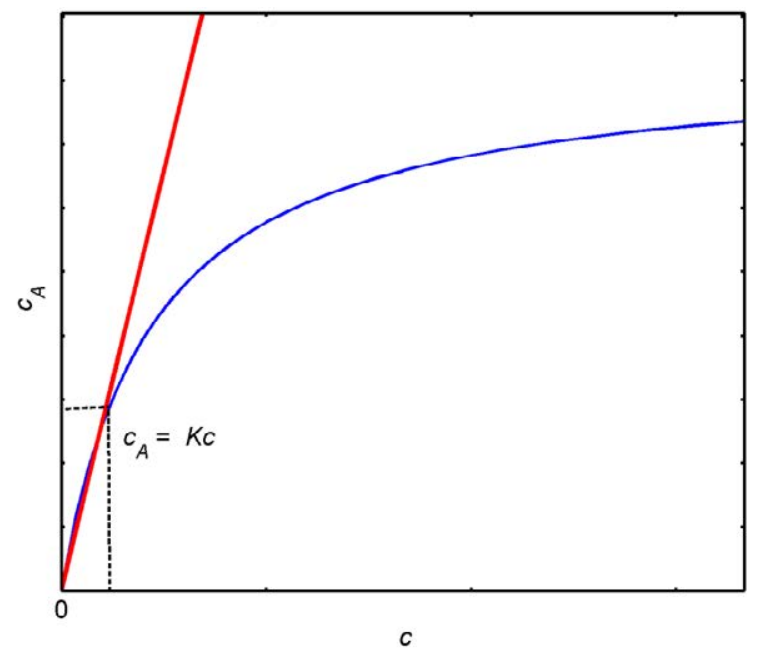

Figure 3. Langmuir adsorption isotherm.

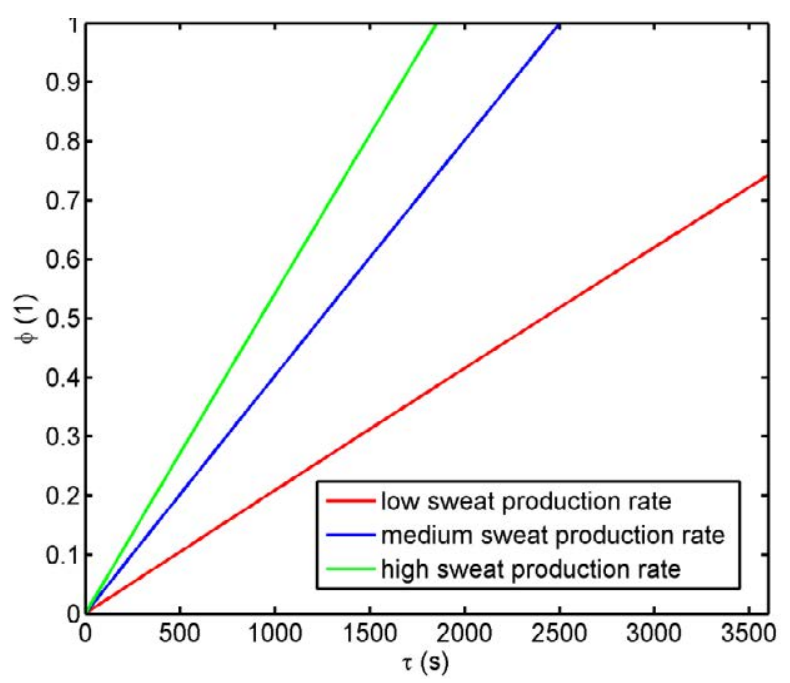

Figure 4. Time dependence of relative humidity concentration in the inner volume of a shoe, shoe upper made of leather with polymer surface treatment, sorption material in the inner shoe volume is present $(K=1000)$. coordination bonds to the collagen protein of hide, thus giving the resulting leather the desired utility properties. However, a small portion of the chromium salt remains unbound and may be partially released in the process of using the tanned goods. From the hygienic point of view, the three valence salts of chromium (Cr III) are healthconscious and do not pose a serious risk to human health. Otherwise, chromate (Cr VI) sixth valence salts are toxic and carcinogenic. The key question is whether trivalent chromium salts can be oxidized to hexavalent chromium salts and under what conditions. Although in most cases the freshly produced leather is free of hexavalent chromium compounds, due to inappropriate use or treatment, oxidation of $\mathrm{Cr}$ III to Cr VI may occur due to the thermodynamic instability of $\mathrm{Cr}$ III salts of chromium [3]. The presence of Cr VI in footwear has been demonstrated several times $[4,5]$. There is potential danger to human health, because hexavalent chromium (Cr VI) salts are easily absorbed by human skin and can cause bladder, kidney and urinary tract cancer [6,7], regardless of dermatological skin disease, as shown in the figure 5.

The best protection is to use socks when wearing footwear, which represent a barrier to the transport of hexavalent chromium to the surface of human skin. The quantitative model of the case is based on the quasi - stationary model presented in figure 6 and is given by the following mass balance - expression (9).

$$
S \frac{c}{2} x(\tau)=D S c \frac{d \tau}{d x}
$$

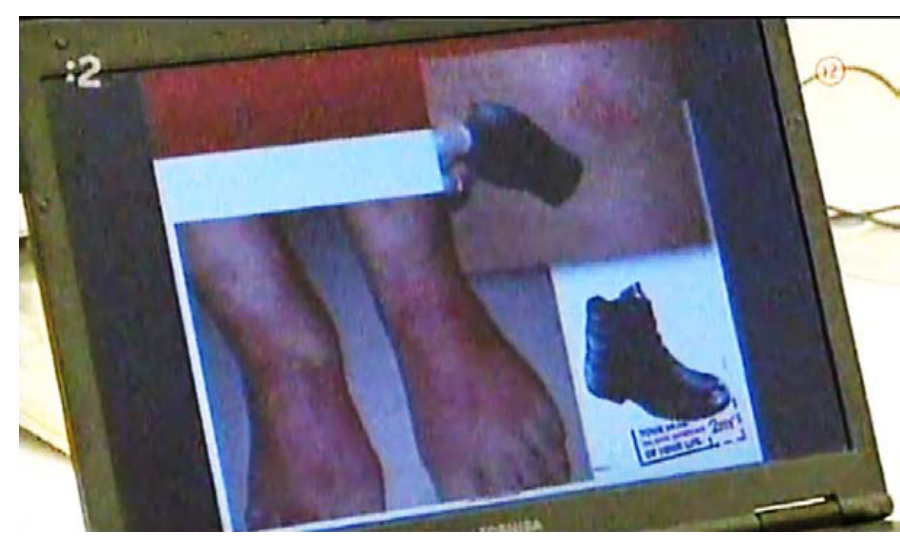

Figure 5. Skin damage caused by compounds of hexavalent chromium contained in shoes leather [9].

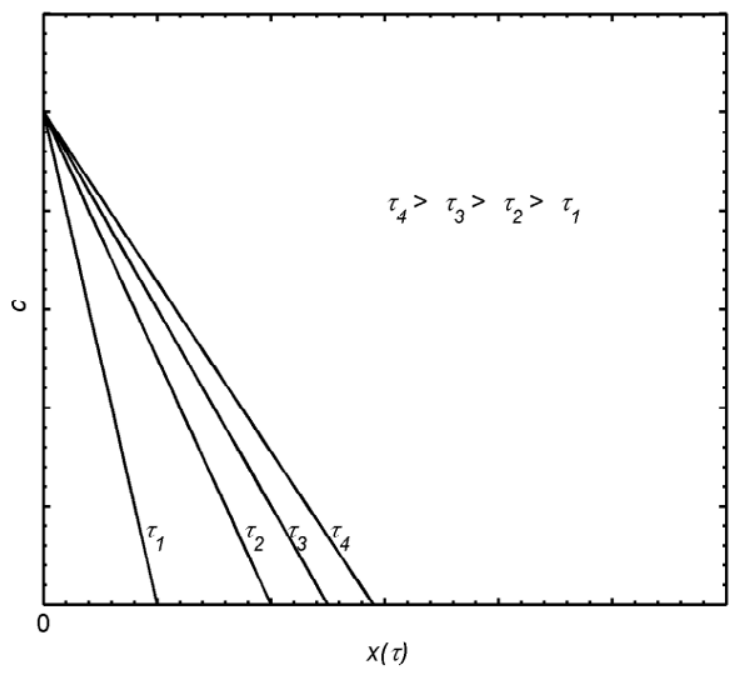

Figure 6. Quasi-stationary model of barrier. 
By integrating the equation (9) we will get:

$$
\tau=\frac{x^{2}}{4 D}
$$

According to equation (10), the safe time is the quadratic function of the sock thickness, when we place the sock thickness after $x$. If it had sorption properties for Cr VI, the diffusion coefficient $(D)$ is divided by $K+1$, where $K$ is the adsorption constant of the linear region of adsorption isotherm. The sock also prevents skin contact for other potentially dangerous components by diffusion adsorption resistance, which may include shoe leather as a result of its manufacture or treatment with various disinfectant agents. Equally important is the protective function of the sock for plastic footwear, which may include migrating plasticizers, especially phthalates (DOP), glue compounds and others, which can cause very unpleasant eczema [8] as shown in figure 7 .

In cities where road transport is dense, toxic poisons resulting from fuel combustion or inappropriate braking (black lines on the road) occur on road surfaces. Even here a sock can play an important protective role.

\section{Conclusions}

Potential damage of foot skin can be caused by unsuitable microclimate in the inner volume of closed shoes and by direct contact of skin surface with upper shoe material, which may contain harmful compounds and next by diffusion or direct contact of skin surface with surrounding environment that contains potential poisons accumulated at the surface of walking roads. The danger of unsuitable microclimate during wearing shoes can be eliminated by a high sorption capacity of upper shoe materials for moisture or by shoe insert. Protection against direct contact of skin surface with potentially dangerous substances like carcinogenic compounds of hexavalent chromium is using socks, which act as a barrier during wearing of shoes. Hexavalent chromium compounds can occur in shoe materials primarily as a consequence of an imperfect technology, especially during tanning operations with chromium compounds or can be formed by wrong treatment of shoes. The treatment of shoes with substances containing oxidants should be avoided (like many commercial disinfectants). As a safety precaution, we recommend to avoid washing dirty shoes using drinking water that contains oxidants in consequence of its disinfection and it is advisable

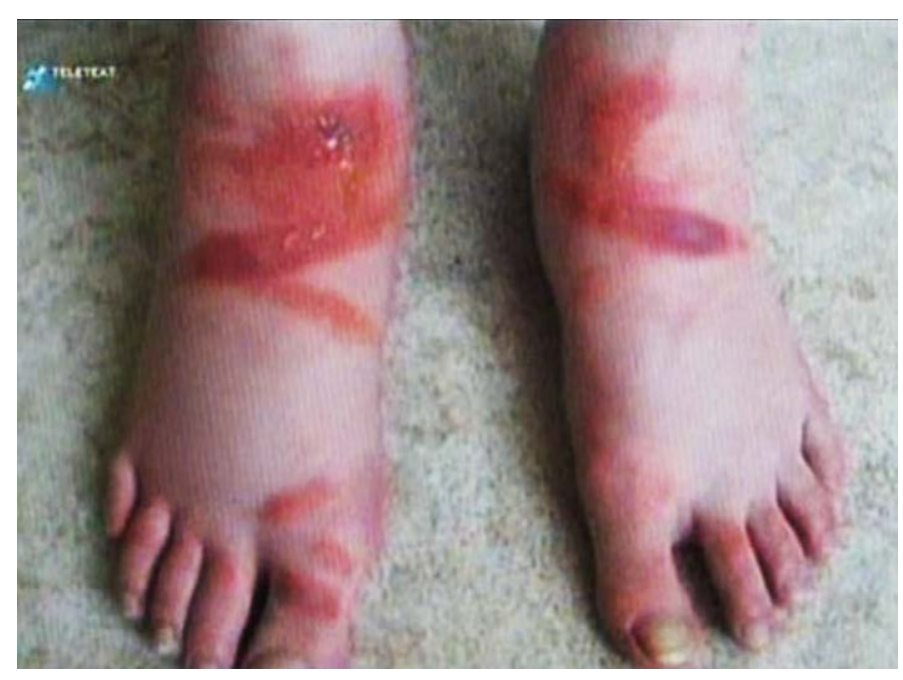

Figure 7. Skin damage by caused by migrating compounds. to treat shoes by suitable reducing cream (acidic creams are the best from this point of view) after wearing them at stormy weather which produces ozone. We may say that wearing of socks can create potential barrier between illness and health or between life and death in the extreme case as conclusion to our paper.

\section{References}

1. Sara A (1986) Thesis, Technical University Brno, Faculty of Technology Gottwaldov.

2. Jirak Z (1975) Correction factor in skin temperature measurement. J Appl Physiol 38: 752

3. Kolomaznik K, Adamek M, Andel I, Uhlirova M (2008) Leather waste--potential threat to human health, and a new technology of its treatment. J Hazard Mater 160: 514-520. [Crossref]

4. Scancar J, Milacic R (2007) Aluminium speciation in environmental samples: a review. Analyt Bioanalyt Chem 386: 999-1012.

5. Karthikeyan R, Ramesh R, Usha R, Ramanaiah B, Chandra NK (2007) Fe (III)-Cr (III) combination tannage for the production of soft leathers. J Am Leather Chemists Assoc 102: $383-392$

6. Hang X, Zhang X, Wang X, Jin L, Yang Z, et al. (2011) Chronic occupational exposure to hexavalent chromium causes DNA damage in electroplating workers. BMC Public Health 11: 224-232.

7. Kirk O (1992) Encyclopedia of Chemical Technology 4thEd. John Wiley \& Sons Inc. New York, USA.

8. Poisonous shoes part 1 (2017) Available from: https://www.youtube.com watch?v=pGUkgYyleWs

9. Poisonous shoes part 2 (2017) Available from: https://www.youtube.com/ watch?v=pGUkgYyleWs
Copyright: (C2017 Kolomaznik K. This is an open-access article distributed under the terms of the Creative Commons Attribution License, which permits unrestricted use, distribution, and reproduction in any medium, provided the original author and source are credited. 